\title{
Un exemple de la traduction comme perversion ou la rencontre de Panero et de Bataille
}

\section{Henry Gil}

\section{(2) OpenEdition}

\section{Journals}

\section{Édition électronique}

URL : http://journals.openedition.org/agedor/728

DOI : 10.4000/agedor.728

ISSN : 2104-3353

Éditeur

Laboratoire LISAA

\section{Référence électronique}

Henry Gil, « Un exemple de la traduction comme perversion ou la rencontre de Panero et de Bataille », L'Âge d'or [En ligne], 7 | 2014, mis en ligne le 01 mars 2014, consulté le 20 avril 2019. URL : http:// journals.openedition.org/agedor/728 ; DOI : 10.4000/agedor.728 
Henry GIL

Université de Paris Est Marne-la-Vallée, LISAA/EMHIS EA 4120

\title{
Un exemple de la traduction comme perversion ou la rencontre de Panero et de Bataille
}

\begin{abstract}
Résumé : Selon Túa Blesa, les traductions de Leopoldo María Panero sont aussi singulières que l'est son œuvre poétique car le poète y revendique une pratique de la traduction comme perversion où le traducteur doit "développer - ou dépasser - l'original et non "le transporter" comme n'importe quel autre meuble d'[une] pièce à l'autre ». Je me propose d'analyser ce concept à travers la traduction d'un sonnet de Georges Bataille, intitulée « Mutación de Bataille ». Comment entendre cette perversion d'un point de vue formel et sémantique sachant que cette rencontre a lieu entre deux écrivains dont l'œuvre se fonde sur la transgression ? Si la traduction-perversion dénature la forme-sonnet à cause de multiples amplifications, elle tire aussi constamment le poème vers l'obscène et l'obsédant retrouvant ainsi une démarche propre aux récits batailliens. Cela se fait grâce à un motif récurrent, absent de l'original, mais qui, ici, semble faire allusion à la stratégie traductrice mise en place, laquelle prétend offrir une «version interlinéaire » du sonnet.
\end{abstract}

Mots-clés : Leopoldo María Panero - traduction - Georges Bataille - sonnet - perversion - trou Walter Benjamin

Resumen: Según Túa Blesa, las traducciones de Leopoldo María Panero son tan singulares como lo es su obra poética pues el poeta reivindica en ellas una práctica de la traducción como perversión en la que el traductor ha de "desarrollar - o superar - el original y no "trasladarlo" como otro mueble cualquiera, de [una] habitación a otra». Me propongo analizar este concepto con la traducción de un soneto de Georges Bataille, titulada "Mutación de Bataille». ¿Cómo ha de entenderse esta perversión desde un punto de vista formal y semántico sabiendo que este encuentro tiene lugar entre dos escritores cuyas obras se fundan en la transgresión? Si la traducción-perversión adultera la forma del soneto a causa de numerosas amplificaciones, también orienta constantemente el poema hacia lo obsceno y lo obsesivo reanudando así con un enfoque propio de los relatos de Bataille. Esto se hace merced a un motivo recurrente, ausente en el poema original, pero que, aquí, parece aludir a la estrategia traductora llevada a cabo, la cual pretende ofrecer una «versión interlineal » del soneto.

Palabras clave: Leopoldo María Panero - traducción - Georges Bataille - soneto - perversión agujero - Walter Benjamin

omme l'explique Túa Blesa, dans sa Préface intitulée «Teoría y práctica de la
traducción como perversión », destinée à introduire Traducciones / Perversiones,
anthologie qui reprend la totalité des traductions poétiques de Leopoldo María Panero, celles-ci sont tout aussi "singulières » que son œuvre poétique. Car pour Túa Blesa, la poésie panérienne en rupture avec «lo creado, el sistema estético, las

\footnotetext{
${ }^{1}$ BLESA, Túa, «Teoría y práctica de la traducción como perversión », in Traducciones/ Perversiones, Anthologie contenant les traductions poétiques panériennes, édition Túa Blesa, Madrid, Colección Visor de Poesía, 2011, p. 7.
} 
convenciones, la idea de lo literario » ${ }^{2}$ est « une écriture de la transgression » qui crée en se fondant sur « un programme de destruction» et un « discours de la violence ». Des données dont il faut tenir compte à propos de la conception panérienne de la traduction car elles nous conduisent à réviser le concept même de fidélité. Ces idées de «transgression » et de « violence » ne supposent-elles pas, en effet, celle de contrevenir à une certaine autorité du texte source, voire de la violer systématiquement ? Comment dès lors peut s'exprimer ce qui devrait être préservé, le ou les sens du poème original par-delà les langues, afin que la traduction reste un poème ? Un poème dont la signifiance provenant à la fois du signifiant au travail, des sens connotatifs et des rythmes est ce qui constitue le difficile enjeu de toute traduction poétique. L'importance donnée par Panero à la traduction révèle un trait propre à la génération novísima, à propos de laquelle José María Castellet dit non sans une volontaire provocation : «su formación literaria es, fundamentalmente, extranjera » ${ }^{3}$.

Le corpus de cette anthologie est constitué de traductions publiées entre 1972 et 1987. On y trouve surtout des traductions d'auteurs anglophones, Edward Lear, Lewis Carroll, John Clare, Robert Browning - poètes pratiquant le nonsense, une folie lucide ou le monologue dramatique - mais aussi Mick Jagger et Keith Richards, auteurs de "Jumpin' Jack Flash ». Panero a également traduit du latin cinq poèmes obscènes de Catulle et du provençal un poème sur le «pur néant» écrit par le troubadour Guillaume de Poitiers. Quant au corpus français, il se limite à un sonnet de Georges Bataille rattaché à L'Archangélique et huit sonnets de Tristan Corbière, appartenant aux Amours jaunes. Quelle que soit la langue du poème original, Panero prétend soumettre ces textes à ce qu'il appelle « une traduction perversion ». Il explique dans «Lo que por fin dijo Benjamin », prologue écrit pour présenter sa première traduction, les Limricks d'Edward Lear: "Que a la traducción cumple desarrollar -o superar- el original, y no "trasladarlo", como otro mueble cualquiera, de esta habitación a otra ». Puis, il précise :

no hay que trasladar, repito, de una lengua a otra, el poema como si fuera un bolso, sino «fundir» las dos lenguas, hacer que se establezca entre ellas un contacto fructífero, y no superfluo como un apretón de manos. ${ }^{4}$

Enfin, dans un autre Prologue, celui de Matemática demente, il désigne sa pratique de la traduction comme « une perversion », terme repris dans le titre de l'anthologie Traducciones/ Perversiones, établie par Túa Blesa et il ajoute :

Ello (ça) habremos de hacer si queremos salvar a un tiempo la letra y el sentido del original (lo que se llamó « espíritu » y «letra »; sólo lo lograremos a costa de ambos, cuando el sentido per-vierta a la letra, y la letra al sentido ${ }^{5}$,

\footnotetext{
2 BLESA, Túa, écrit, dans la préface intitulée «La destruction fut ma Béatrice », qui ouvre le 1er Tome de Poesía Completa, celui de la période (1970-2000), pour présenter l'œuvre panérienne : «Y esta obra se hace precisamente quebrando página a página, libro a libro, lo creado, el sistema estético, las convenciones, la idea de lo literario. $\mathrm{O}$, dicho de otro modo, esta obra, una escritura de la transgresión, crea, dice, no ya lo que se viene entendiendo por literatura, sino qué es lo que pueda llegar a ser tenido por literario, siendo así toda una auténtica exploración ». Madrid, Colección Visor de Poesía, 2000, p. 7-8.

3 José María Castellet, Nueve novísimos poetas españoles, réédition de l'anthologie de 1970, Barcelona, Ediciones Península, 2001, p. 39.

${ }^{4}$ Cette traduction des Limricks d'Edward Lear, parait en édition bilingue sous le titre El ómnibus, sin sentido, Madrid, Visor, Alberto Corazón editor, 1972. Cette réflexion de Panero fait partie de son prologue, p. 7-8.

${ }^{5}$ PANERO, Leopoldo María, dans le prologue écrit pour sa traduction des textes de Lewis Carroll, Matemática demente, Barcelona, Tusquets editores, p. 18-19.
} 
le trait d'union entre « per» et « vierta » suggérant sous la forme d'un jeu de mots la lecture d'une « version à travers ».

Je pensais porter mon attention sur l'ensemble du corpus français, mais il m'a finalement paru préférable de ne m'en tenir qu'à la «traduction-perversion » du poème de Bataille, que Panero a, d'ailleurs, appelée, assumant la transformation de ce sonnet: «Mutación de Bataille » et qu'il a insérée dans la section "Palimpsestos » qui vient clore Narciso en el acorde último de las flautas. En quoi réside réellement cette perversion, quel sens lui donner, comment l'entendre d'un point de vue formel et sémantique par rapport à l'original ?

Pour justifier la place de ces traductions au sein du recueil, Túa Blesa rappelle que précédemment figurent, dans la section "Matrimonio de las cenizas », une citation de Bataille, tirée de L'Archangélique ${ }^{7}$, traduite en espagnol, et une brève allusion à Corbière au sein du poème «Descort ». Mais, il ne dit pas que cette citation traduite s'écarte, elle aussi, légèrement de l'original en rajoutant un mot, puisque nous avons : " $\mathrm{El}$ no-amor es la verdad del amor/ y todo miente en la ausencia de amor $»^{8}$ pour : «Le non-amour est la vérité/ et tout ment dans l'absence d'amour ». Ce rajout ne change pas fondamentalement le sens de ces vers, mais il les rend sans doute plus incisifs en soulignant le paradoxe grâce à une épiphore. En fait, la présence de Bataille est probablement plus importante que ne le laissent croire cette citation et ce sonnet tous deux traduits car si L'Archangélique comprend trois sections qui sont « Le tombeau », «L'aurore » et «Le vide », Narciso en el acorde último de las flautas en comporte sept dont la première s'intitule «Luz de tumba » et la troisième "Para hacer el vacío", autrement dit un même champ lexical et une même thématique imprègnent les deux recueils. Et lorsque Panero intitule en français un poème " Ma mère » comment ne pas penser au récit du même nom écrit par Bataille?

Quand on compare les poèmes originaux du corpus français et leur traduction, on comprend tout de suite, ne serait-ce que visuellement, comment s'est mise en place la «perversion ». Ces neuf poèmes sont des sonnets, donc des formes clairement délimitées et obéissant toutes de façon très stricte au genre auquel elles appartiennent (nombre de vers, types de mètres et de strophes, schéma des rimes). Or, non seulement les traductions de Panero ne respectent ni les mètres ni les rimes des originaux mais, parfois, elles ne tiennent même pas compte de la disposition typographique des strophes inhérentes au sonnet et surtout nous proposent des textes qui dépassent largement les quatorze vers propres à la forme-sonnet comme celle du sonnet de Bataille qui compte 22 vers. Avant de voir comment fonctionne la "perversion» effectuée par la traduction du sonnet de Georges Bataille, il faut essayer de cerner la signification et donc les différentes pistes qui pouvaient s'offrir au traducteur « pervers ».

Je rêvais de toucher la tristesse du monde au bord désenchanté d'un étrange marais je rêvais d'une eau lourde où je retrouverais les chemins égarés de ta bouche profonde

\footnotetext{
${ }^{6}$ Ce recueil publié chez Visor, en 1979, est réédité dans Poesía Completa (1970-2000), Madrid, Visor de Poesía, 2010, p. 137-203. La dernière section ne compte que des traductions, ce sonnet de Bataille, des sonnets de Tristan Corbière et la traduction d'un poème de John Clare.

${ }^{7}$ BATAILLE, Georges, L'Archangélique et autres poèmes, Préface Bernard Noël, Paris, Poésie/Gallimard, En fait ce sonnet sans titre est rangé dans une section à part, qui n'a pas été retenue pour L'Archangélique et que Bataille appelle «Appendice (Poèmes disparates) », p. 100. Le sonnet est page 102 de cette édition.

${ }^{8}$ Cette citation de Bataille traduite par Panero et qui ouvre «El matrimonio de las cenizas », la 4e section de Narciso en el acorde último de las flautes, se trouve p. 163, in Poesía Completa (1970-2000), op. cit.
} 


\begin{abstract}
j'ai senti dans mes mains un animal immonde
échappé à la nuit d'une affreuse forêt

et je vis que c'était le mal dont tu mourais

que j'appelle en riant la tristesse du monde

une lumière folle un éclat de tonnerre

un rire libérant ta longue nudité

une immense splendeur enfin m'illuminèrent

et je vis ta douleur comme une charité

rayonnant dans la nuit la longue forme claire

et le cri de tombeau de ton infinité.
\end{abstract}

Ce qui frappe d'emblée dans ce sonnet et qui peut expliquer qu'il ait été écarté par Bataille du recueil L'Archangélique, c'est - bien qu'il partage un lexique et des thèmes communs à celui-ci - le classicisme de son écriture. Car, la quasi-totalité des textes de ce recueil est composée de brefs poèmes aux vers libres, plutôt courts et jamais rimés. Ici, dans ce sonnet, nous avons, en revanche, de très classiques alexandrins binaires $(6 / 6)$ aux rimes embrassées dans les quatrains (ABBA) et au schéma français (CDC/DCD) dans les tercets, alternant rimes féminines et masculines, excepté lors du passage des quatrains aux tercets. Dans le reste du recueil, les vers brefs, souvent distribués en tercets, parfois en quatrains, plus rarement en distiques, se succèdent comme des éclats au sein d'une scansion parataxique sans autre ponctuation que le point final qui clôt chaque poème comme dans ce sonnet, le tout suggérant la présence lyrique et tourmentée d'un moi angoissé, sorte d'ange déchu, animé d'un désir de l'autre qui semble ne pouvoir s'accomplir que dans la mort. La forme-sonnet confère au poème un cadre beaucoup plus harmonieux, voire serein, empreint d'une certaine musicalité bien que les thèmes du poème recoupent ceux des autres textes du recueil. Il s'agit d'une rêverie érotique du Moi (" je rêvais ») sur le corps de la femme aimée comme le suggère la fin du premier quatrain (" ta bouche profonde »), mais un corps d'abord envisagé de façon énigmatique et paradoxale, ( "la tristesse du monde », "eau lourde»), et qui semble être aussi un espace géographique malsain, où règnent des eaux stagnantes où l'on risque de s'enliser sans illusion (" un étrange marais »). Cependant l'anaphore " je rêvais » montre le caractère obsessionnel de ce désir. Un désir qui est tendu, comme toujours chez Bataille, par un radical renversement des valeurs. Et c'est ce qui explique que, chez lui, le champ lexical du mal se substitue à celui du bien. Un mal qui se manifeste à la fois comme tristesse, souffrance, saleté, obscénité, puis dans le quatrain suivant, comme animalité, férocité mais dont le but unique est de retrouver "les chemins égarés de ta bouche profonde ", autrement dit la voie d'accès à l'amour humain, à l'Éros, présente dans presque tous les poèmes d'amour, envisagée ici comme une pénétration dans le corps de l'autre comme le suggère le qualificatif « profonde ».

Dans le deuxième quatrain, le Moi évoque l'étreinte avec le corps aimé, décrit comme une animalité à la fois attirante et répugnante (" un animal immonde »), issue d'un espace obscur, sauvage et primitif (" échappé à la nuit d'une affreuse forêt ») et sans doute horrible qui renvoie à la mort (" c'était le mal dont tu mourais »). L'amour est envisagé comme un «mal » qui est une permanente agonie comme le suggère l'imparfait (« dont tu mourais ») tandis que le Moi semble s'y référer avec sarcasme ("que j'appelle en riant la tristesse du monde »), signalant qu'est atteint cet objet tant convoité, nommé dès le premier vers du 
poème comme «la tristesse du monde », expression qui renvoie à un état affectif supposant la peine, le malaise tout en lui attribuant une dimension cosmique. L'expression délictueuse propre à Bataille de la violence, de l'excès, de la transgression, du rejet des conventions et des normes ne pouvait que plaire à Panero. Le langage poétique de Bataille, conçu comme «le couteau du boucher dans la langue »" naît de la "haine de la poésie » comme le suggérait l'ancien titre d'un de ses livres ${ }^{10}$. Il y a chez lui un profond rejet de la «belle poésie » fondée sur le bon mot, sur la niaiserie sentimentale et l'évanescence lyrique; tout ce qui pourrait correspondre au bon sens, au bon goût, voire à un idéal qui prétend accéder à un savoir alors que la mort même devrait rappeler à l'homme qu'il est confronté au nonsavoir, car le savoir «ne permet pas de penser la mort», la mort qui exerce sur lui une fascination. Or, c'est justement cet innommable que Bataille veut regarder en face, lui qui déclare : «Ma propre mort m'obsède comme une cochonnerie obscène et par conséquent horriblement désirable ${ }^{11}$. Il y a donc dans son écriture une volonté, par le biais de véritables "pulsions verbales » en rupture avec ce qui est convenu, de choquer, pour éveiller en chacun une inquiétude, la sensation d'une précarité, la peur même de la mort, avec laquelle l'érotisme qui, selon lui, pourrait être «l'approbation de la vie jusque dans la mort $»^{12}$ lui apparait comme le «meilleur moyen de se familiariser avec (elle) $»^{13}$. Et pour fuir toute «l'écœurante sentimentalité poétique »" Bataille, comme le dit très justement Bernard Noël « déchante et désenchante afin de réduire le poème à une nervosité excessive, à l'à-vif » ${ }^{15}$.

Le premier tercet exprime le ravissement extatique de cette rencontre du Moi avec le corps nu de l'être aimé ( " ta longue nudité ») par une juxtaposition d'expressions dont trois ("une lumière folle un éclat de tonnerre/ une immense splendeur») renvoient au sème /lumière/ et sont sujets d'un verbe réitérant également le même sème ("m’illuminèrent»). Cette gradation ascendante exprime une montée orgasmique, celle de «la petite mort». Au sein de cette gradation surgit la manifestation d'une gaieté libératrice (" un rire libérant»), déjà amorcée lors du dernier vers du deuxième quatrain avec la formule oxymorique ("que j’appelle en riant la tristesse du monde ») et sans doute rappelé ici par le mot "éclat» applicable autant au « rire » qu'au « tonnerre ».

Le dernier tercet est la conséquence du précédent surdéterminé par le sème /lumière/ qu'il reprend ici avec le verbe (« rayonnant»). L'anaphore (« et je vis »), écho du vers 7, mais, dans un sens, désormais, clairement «oculaire» souligne l'importance du regard porté sur le corps de l'aimée. Un regard supposant, comme toujours chez Bataille, un renversement des valeurs. Car là où on attendrait le terme "plaisir", Bataille nous propose le mot « douleur», comme au vers 1 «la tristesse » était l'envers de «l'allégresse ».

\footnotetext{
9 À propos de la conception bataillienne de la poésie, Michel Surya écrit dans Georges Bataille, la mort à l'cuvre : « la poésie n'est la poésie que si elle est un délit, si elle a le caractère de lubricité du déchaînement sexuel : que si elle est un crime : le couteau du boucher dans la langue (belle, noble, élevée). » SURYA, Michel, Georges Bataille, la mort à l'cuvre, Paris, Tel, Gallimard, 2012, p. 377.

10 BATAILLE, Georges, L'impossible, in CEuvres complètes III, Paris, Gallimard, 2008. Ce livre fut publié, en 1947, aux Éditions de Minuit, sous le titre La haine de la poésie, puis réédité chez Gallimard, en 1962, avec son titre actuel.

11 Phrase de Georges Bataille rapportée par Bernard Noël, dans sa préface, intitulée « Le bien du mal », écrite pour son édition de BATAILLE, Georges, L'Archangélique, op. cit., p. 11.

${ }^{12}$ L'introduction écrite par Bataille pour son essai L'érotisme, Paris, Les éditions de Minuit, 1957, s'ouvre sur la phrase suivante : «De l'érotisme, il est possible de dire qu'il est l'approbation de la vie jusque dans la mort ». p. 17.

13 Ibid., p. 31.

14 Titre donné par Michel Surya à l'un des chapitres de son essai Georges Bataille, la mort à l'cuvre, op. cit., p. 372.

${ }^{15}$ NOËL, Bernard, dans « Le bien du mal », préface écrite pour L'Archangélique et autres poèmes, op. cit., p. 8.
} 
Le voyeurisme mis à l'œuvre n'est évidemment pas exempt d'une certaine forme de sadisme où la «douleur» de l'autre est offerte comme un don d'amour ("comme une charité »), expression volontairement sacrilège puisque la « charité » est une vertu théologale qui «consiste dans l'amour de Dieu et du prochain en vue de Dieu». Or, ici la seule divinité c'est le corps de l'autre, livré à une extase le transfigurant et qui pourrait être tout aussi bien sa mort («le cri du tombeau»), mais une mort qui, bien qu'impliquant la limite, semble se charger d' "infinité », domaine propre à Dieu. L'emploi de l'anacoluthe liée au verbe « rayonnant» permet au fil de la lecture trois interprétations successives destinées à chaque fois à surprendre. En effet, une première lecture jusqu'à la fin du premier hémistiche du vers 13 , suggère que le complément d'objet direct «ta douleur » devient sujet de «rayonnant». Cependant, le deuxième hémistiche du vers 13 indique une nouvelle lecture où le sujet de "rayonnant» est désormais «la longue forme claire » avant que le dernier vers ne permette de comprendre qu'il faut ajouter un nouveau sujet («Le cri de tombeau de ton infinité »). Une fois de plus Éros et Thanatos sont donc liés.

Voyons à présent de quelles façons s'est exercée sur ce sonnet la traductionperversion de Panero afin d'en cerner toute sa singularité.

\section{MUTACIÓN DE BATAILLE}

(De L'Archangélique)

Yo soñé con tocar la tristeza viscosa del mundo en el desencantado borde de una ciénaga absurda yo soñé un agua turbia donde reencontraría el camino perdido de tu ano profundo;

yo he sentido en mis manos un animal inmundo que en la noche había huido de una espantosa selva salvaje como el viento, como el negro agujero de tu cuerpo que me hace soñar yo he soñado en mis manos un animal inmundo y supe que era el mal del que tú morirás y lo llamo riéndome del dolor del mundo.

Una demente luz, una luz que hace daño encuentra sólo en mí el cadáver de tu risa de tu risa que libra tu larga desnudez y el viento descubre nuestra muerte, semejante a ese agujero inmundo que yo quiero besar; un resplandor inmenso entonces me iluminará y he visto tu dolor como una caridad irradiando en la noche tu forma amplia e inmensa el grito de la tumba que es tu infinidad

$\mathrm{y}$ he visto tu dolor como una caridad, como si alguien dejara suavemente un ojo en la mano blanca que un mendigo le tiende. 
Avec 22 vers, le poème traduit se trouve rallongé d'un peu plus d'un tiers. La «mutation» suppose toutes sortes d'excroissances qui touchent d'abord le lexique, puis progressivement le mètre et la strophe. Ainsi, du point de vue typographique, seule, la première strophe demeure un quatrain, car le quatrain suivant compte, lui, 7 vers et aux deux tercets correspond une seule et longue strophe de 11 vers, où l'on voit que le premier tercet donne lieu à 6 vers, et le second à 5 . Panero n'essaye pas de conserver les rimes excepté lorsque la traduction littérale les amène naturellement («mundo », "profundo», «inmundo»). Quant aux mètres, Túa Blesa dirait que, comme dans la plupart des traductions-perversions, ce sont des vers libres. Cependant, 10 d'entre eux sont de véritables alejandrinos (vv3/4/5/7/9/10/12/14/18/19) et parmi eux les vv7 et 12 sont une création originale de Panero. Par ailleurs, les vers 16 et 20, de vingt et une syllabes sont des alejandrinos et demi. Cependant, si la moitié des vers sont d'authentiques alejandrinos, la plupart des autres sont des alejandrinos boiteux, trop longs ou trop courts. Seuls les vers 8 et 17, respectivement déca et ennéasyllabes, semblent échapper à la mémoire de l'alejandrino que la traduction altère ou pervertit de façon significative.

Les altérations dues à des rajouts, parfois un mot, à d'autres moments plusieurs, ou à des substitutions de mots, viennent changer le sens. Les deux procédés sont propres à toutes les traductions de Panero quelle que soit la langue du texte source. Le premier renvoie, selon Túa Blesa, à un procédé rhétorique qui est l'amplification qui consiste «à développer des idées par le style, de manière à leur donner plus d'ornement, plus d'étendue ou plus de force $»^{16}$. C'est un procédé systématique et toujours quantifiable. Il correspond à la conception panérienne de la traduction, selon laquelle cette dernière doit " développer », ou «dépasser l'original et non le transporter comme un meuble d'une pièce à l'autre ». Nous reviendrons plus tard sur les raisons invoquées pour justifier une telle démarche qui n'a pas manqué de s'attirer maintes critiques et qui compte peu de précédents, excepté celui des traductions d'Ezra Pound.

Il est intéressant d'observer que dans le premier vers, Panero pervertit volontairement le rythme de l'alejandrino en rajoutant à «tristeza «le qualificatif «viscosa » ce qui donne un heptadécasyllabe $(7 / 10)$ alors que la même traduction sans ce rajout eût donné un parfait alejandrino tétradécasyllabique équivalent de l'alexandrin dodécasyllabique français (6/6). L'adjectif «viscosa» est le seul rajout de cette strophe, en revanche, la perversion va consister à introduire, toujours à dessein, des variantes qui sont des faux sens plus ou moins importants (" absurda » pour « étrange », «turbia » pour «lourde » et surtout «ano» pour «bouche »). Le rajout du qualificatif « viscosa» permet de rendre, d'emblée, plus présente et plus concrète, une sensation associée au toucher, lequel est invoqué chez Bataille comme objet de la rêverie. Mais, évidemment, ce rajout fonctionne aussi comme une clarification, destinée à rendre évident ce qui serait celé ou réprimé dans l'original bien qu'en fait cela ne puisse apparemment se déduire que des vers 3 et 4 («marais » et « eau lourde ») et ne sera évident qu'au vers 5 avec le qualificatif «immonde ». Chez Panero l'objet de la rêverie marquée par le champ lexical de la mélancolie ("tristeza», "desencantado », " perdido ») présent dans l'original se voit tirer d'emblée vers celui de la saleté, de la souillure, du cloaque, voire de l'excrémentiel (« viscosa », « turbia », « ano »). Ce monde du «visqueux » n'étant, en fait, implicite dans le quatrain de l'original que dans le mot "marais », qualifié d' "étrange» comme pour en atténuer les possibles connotations négatives (enlisement, insalubrité, odeur nauséabonde) alors que le qualificatif " absurda » indique clairement, lui, la privation de sens. Túa Blesa se contente d'indiquer que la substitution de «ta bouche » par «tu ano » répond à la thématique et au lexique propre à

${ }^{16}$ DUPRIEZ, Bernard, Gradus. Les procédés littéraires (Dictionnaire), Paris, 10/18, nº 1370, 1984, p. 41. 
Panero. Cette substitution si elle renvoie sans nul doute à l'homosexualité du locuteur panérien et à un corps aimé masculin ne peut se limiter à cela car elle n'en reste pas moins significative dans la stratégie traductrice panérienne qui est de tirer à chaque fois plus le poème vers l'obscène, vers ce qui ne doit pas se dire ou se montrer et qui agit de façon obsédante sur le Moi. Car l'anus fonctionne dans la traduction-perversion comme un point de fixation obsessionnelle pour le locuteur panérien qui le définit ensuite comme «negro agujero » quand il le fait réapparaittre aux vers 7 et 8 , rajouts sans lien apparent avec le sonnet de Bataille. L'expression «negro agujero » rappelle le concept en astronomie de trou noir qui désigne un «objet théorique extrêmement dense exerçant une attraction si forte qu'il n'émet aucun rayon ». La métaphore panérienne suggérant ainsi, au milieu de la strophe, la force d'attraction de ce motif.

Le vers 5, parfaite traduction littérale de l'original, préserve l'alejandrino: "Yo he sentido en mis manos/ un animal inmundo» (14=7/7). Le qualificatif «inmundo» complète ainsi le champ lexical de la saleté présent dans la traduction-perversion. Une saleté qui s'identifie à la sexualité car c'est de cette façon que la qualifie l'ordre moral lorsqu'elle s'effectue en dehors de la procréation: "le sexe, c'est sale », sans oublier l'importance en elle de l'animalité, autrement dit des pulsions instinctives. Enfin, la rêverie se fait chair donnant lieu à une sensation tactile. La traduction du vers 6 connaît une légère variation faisant de la nuit plus un moment dissocié de "l'affreuse forêt» qu'un univers faisant corps avec elle et participant à son énigmatique et sombre univers. Puis une nouvelle amplification est amorcée grâce au qualificatif « salvaje » appliquée à la " espantosa selva », qualificatif amené par l'image de l'animal associé à son habitat, une forêt qui dans la traduction ne peut correspondre qu'à la jungle, univers de la férocité et de la sauvagerie. L'amplification s'exprime, ici, par le biais d'une double comparaison totalement absente dans l'original où le comparé « espantosa selva » reçoit un double comparant « el viento »symbole d'agitation, de force élémentaire - et « el negro agujero/ de tu cuerpo » qui non seulement fait ressurgir « l'anus » mais le présente comme l'objet jusqu'alors énigmatique et caché de la rêverie du Moi (" que me hace soñar»). La simple variante «ano » au lieu de «bouche » est donc présentée comme l'instigatrice de la rêverie, d'où la quasi réitération au vers 9 du vers 5 , mais en lui associant le verbe «soñar» du premier quatrain comme si le locuteur abandonnant l'étreinte retournait à sa rêverie. Un vers 9 inédit qui, néanmoins, préserve le mètre de l'alejandrino. Quant aux vers 10 et 11 correspondant aux vers 7 et 8 de l'original, ils connaissent également des variations allant pour le dernier jusqu'au contresens. Car le vers 10 transforme la vision en savoir et annonce la mort de l'autre, non à l'imparfait, temps qui se prolonge dans le passé mais au futur, temps prophétique ("y supe que era el mal del que tú morirás »). Et si le vers 8 dans le sonnet français consiste à revenir sur «la tristesse du monde » pour nous en révéler sa nature - celle qui conduit l'autre vers une mort qui semble être son accouplement avec le Moi -, le vers 11 panérien transforme, lui, la phrase en changeant le sens du verbe «appeler» puisque «llamar» ne renvoie plus à la façon de nommer le «mal d'amour» mais au fait de le convoquer, tout en se moquant de la "douleur du monde », le mot «dolor » s'étant substitué à celui de «tristeza », attitude de défi du locuteur panérien vis-à-vis de l'univers. On peut observer que le premier hémistiche tel qu'il a été traduit : "y lo llamo riéndome », s'il avait été suivi d'une traduction littérale en espagnol : "la tristeza del mundo", aurait donné un parfait alejandrino au lieu d'un tridécasyllabe (7/6). On voit donc bien la volonté chez Panero de jouer constamment avec l'alexandrin et de le rendre bancal.

Alors que dans l'original, il n'y a un point qu'à la fin du dernier vers du sonnet, ici, l'on en trouve un avant ce qui s'annonce comme la traduction des tercets, laquelle commencée par un léger retrait se présente comme une amplification croissante sur 11 
vers, la deuxième partie de la traduction ayant exactement le même nombre de vers que la première contrairement à ce qui se produit dans un sonnet. Dans l'original, le premier tercet disait la montée orgasmique grâce au champ lexical de la lumière. Or, l'on ne retrouve plus, à présent, dans la traduction, à cause de l'amplification, l'impression d'une gradation croissante due à une juxtaposition d'éléments pour la plupart lumineux et tous sujets du verbe «m'illuminèrent». L'amplification commence avec la caractérisation de la «lumière » qui n'est pas seulement «demente», donc passionnée et irraisonnée, mais facteur de souffrance chez le Moi et en partie morbide puisqu'elle ne trouve que «el cadáver de tu risa ». Or, même si Bataille a constamment recours au renversement des valeurs pouvant suggérer ainsi suivant un schéma sadomasochiste que la « douleur » signifie aussi le "plaisir », dans ce tercet rien ne s'exprime selon cette inversion, où un mot négatif y ferait entendre son contraire. En revanche, chez Panero le mot «cadáver» qui suppose « un corps mort» s'applique ici par une sorte d'hypallage au "rire » (" el cadáver de tu risa ») qui dans la traduction panérienne devient donc celui de l'être désiré. L'amplification se poursuit grâce à une anadiplose (" de tu risa/ de tu risa que libra tu larga desnudez »). On retrouve la nudité du corps de l'autre, nécessaire pour qu'il y ait union entre les amants, mais elle s'exprime ici sur le mode d'une mort partagée (" nuestra muerte »), révélée par le vent, force élémentaire qui semble dévoiler l'issue de cette relation qui renverra à la mort des amants («y el viento descubre nuestra muerte »). Une fois de plus l'amplification se poursuit, sous la forme d'une comparaison dont le comparant renvoie au motif panérien de la rêverie, motif obsédant qu'est cet « agujero inmundo » qu'il veut embrasser. L'anus décrit comme «agujero inmundo » sert de comparant à la mort crainte et désirée dont Bataille dit qu'elle "(l)'obsède comme une cochonnerie obscène et par conséquent horriblement désirable ». Contrairement au tercet qui exprimait sur le mode d'une gradation ternaire le ravissement extatique, celui-ci n'apparaît qu'à la fin du $16^{\mathrm{e}}$ vers - cet alejandrino et demi avec un seul sujet et un futur, comme si le locuteur était encore dans l'attente de cet instant fatidique et hautement désiré. Or, s'il s'agit de sa vraie mort, il est, en effet, encore dans l'attente de ce moment auquel il semble aspirer parce qu'il le fascine tout autant que Bataille, le "trou noir» correspondant, bien évidemment, aussi à la cavité obscure du «tombeau ». Tout le recueil panérien, par les titres des sections ou des poèmes, renvoie à la mort («Luz de tumba», "Pavane pour un enfant défunt», "Cómo escribía antes de matarme ", "glosa a un epitafio », "Dead flower to a worm », "El matrimonio de las cenizas », « Cópula con un cuerpo muerto », «Un cadavre chante »).

Cependant, le premier vers du dernier tercet, traduit littéralement, propose un nouvel alejandrino : "Y he visto tu dolor como una caridad » $(14=7 / 7)$ comme si l'union entre le Moi et le corps de l'autre avait réellement eu lieu puisqu'il en résulte une vision, laquelle peut, néanmoins, supposer un événement à venir, la mort du sujet évoquée comme une extase amoureuse au sein d'un acte sadomasochiste. Dans les vers 18/ 19/ 20, correspondant à l'anacoluthe du premier tercet, nous retrouvons un usage de la syntaxe se prêtant à différentes interprétations au fil de la lecture, les deux premières sont identiques à celles observées dans l'original, en revanche, la troisième fait de «tu forma amplia e inmensa » le sujet de la proposition gérondive et de « el grito de tu tumba », le complément d'objet direct du verbe "irradiando", alors que celui-ci apparait comme un second sujet dans l'original. Si le sens change légèrement, l'effet de surprise au fil de la lecture demeure et la synesthésie (vue/ son) est préservée. On peut aussi observer la non-traduction du qualificatif «claire » appliquée à la «forme » de l'autre, au profit d'un doublon redondant «forma amplia e inmensa ». Contrairement à l'écriture de Bataille fondée sur la fulgurance, celle de Panero, soumise entièrement à l'amplification, se fonde sur la dilatation et la répétition. 
Comment s'étonner alors de voir, pour conclure, la réitération, aux vers 20 et 21 , du vers 18: "y he visto tu dolor» afin d'introduire une nouvelle amplification totalement absente de l'original : " como si alguien dejara suavemente/ un ojo en la mano blanca que un mendigo le tiende ». Panero reprend ici l'idée de la charité appliquée à la douleur qui comme un «don» serait offerte par l'être désiré au Moi, mais en y ajoutant l'image de l'aumône faite à un mendiant et en remplaçant la pièce de monnaie par un œil. L'œil, support de la pulsion scopique donne accès à l'obscénité et il est évidemment lui-même ce «negro agujero», ce sombre « œillet» ou «ojete» qu'exhibe de façon impudique la blancheur de la main. Quel sens donner à cette amplification? Encore une fois, l'amplification est là pour apporter une image seconde et inédite qui vient gloser l'image première bataillienne. Bien que ces vers soient étrangers au sonnet, la référence à Bataille est plus qu'évidente. Il suffit de penser au titre d'un de ses célèbres récits Histoire de l'œil, où l'écrivain passe en revue les sommets de son obscénité personnelle. Une obscénité qui est une quête se présentant comme une tension permanente vers l'« Impossible », mot qui servit de titre à un récit intitulé précédemment La baine de la poésie. Cette obscénité érigée en spectacle dérangeant consiste en une mise en scène du corps et du langage révélant des situations désavouées par la communauté, car transgressives à l'égard des bienséances et de l'ordre établi en choisissant le sexe et la mort comme terre d'élection. Or, l'œil, organe à l'origine de la pulsion scopique, est l'agent même de l'obscénité et va, dans Histoire de l'ail, jusqu'à l'incarner lorsqu'il sera celui du prêtre violé, assassiné puis énucléé par Simone, qui finira par insérer l'œil du martyr dans sa vulve. Un œil qui, chez Bataille, se focalise sur la dégradation, fin première de l'obscénité car pour lui « il n'est pas de forme répugnante dont (il) ne discerne l'affinité avec le désir ${ }^{17}$. L'obscénité bataillienne c'est donc l'œil à l'œuvre face au sexe et à la mort, un œil fasciné par l'horreur, autrement dit par ce que la société tient secret: le sexe et la mort. Cette dernière amplification insiste sur le pouvoir de l'œil qui fait du Moi voyeur un mendiant implorant de façon obsédante le spectacle fascinant pour lui de l'obscénité auquel l'être désiré répond avec amour et douceur.

Conscient que sa conception de la traduction trouverait des contradicteurs, Panero a essayé de la justifier dans «Lo que dijo por fin Benjamin », en se fondant, en partie, sur La tâche $d u$ traducteur $^{18}$ de Walter Benjamin, essai à la fois complexe et sibyllin sur la traduction car susceptible de diverses interprétations. Pour le philosophe allemand, si la traduction "procède de l'original», cette corrélation dépend, en fait, "moins de sa vie que de sa « survie » car la traduction vient après l'original ». Or, pour Benjamin cette «survie » est forcément, grâce à la traduction, un "renouveau». Ainsi écrit-il à propos des traductions : «En elles, la vie de l'original dans son constant renouveau connait son développement le plus tardif et le plus étendu $»^{19}$. Et il ajoute : «aucune traduction ne serait possible si son essence ultime était de vouloir ressembler à l'original. Car dans sa survie, qui ne mériterait pas ce nom si elle n'était pas mutation et renouveau du vivant, l'original se modifie $»^{20}$.

La «tâche du traducteur» consiste donc «à faire mûrir [...] la semence du pur langage $»^{21}$. Pour Walter Benjamin, les langues ne seraient pas totalement étrangères les unes aux autres et il existerait donc entre elles un rapport intime et caché qu'il serait

\footnotetext{
17 Réflexion faite par Bataille dans sa préface à Madame Edwarda, roman publié en 1941, sous le pseudonyme de Pierre Angélique, repris dans BATAILLE, Georges, Euvres complètes III. CEuvres littéraires, Paris, Gallimard, 1971, p. 11.

${ }^{18}$ BENJAMIN, Walter, La tâche du traducteur, in CEuvre I, Folio essais, Paris, Gallimard, 2000, p. 244-262.

${ }^{19}$ Ibid., p. 246-248.

${ }^{20}$ Ibid., p. 249.

${ }^{21}$ Ibid., p. 255.
} 
possible à une traduction non de créer mais de « représenter en le réalisant en germe ». On voit bien ce qui chez Benjamin peut intéresser Panero. La traduction est une mutation nécessaire qui condamne la littéralité et qui semble autoriser une liberté qui va bien au-delà de la simple «transposition créatrice» défendue par Roman Jakobson à propos de la traduction poétique. C'est pourquoi, selon Walter Benjamin: «la traduction touche l'original de façon fugitive et seulement dans le point infiniment petit du sens, pour suivre ensuite sa trajectoire la plus propre, selon la loi de la fidélité dans la liberté du mouvement langagier $»^{22}$.

Or Panero reprend cette image de la tangente, quand il écrit: "traducción y traducido no deben ser paralelas, sino una tangente (la traducción) que toca el círculo (lo traducido) $»^{23}$. Mais, cela justifierait-il pour autant de farcir constamment, comme nous l'avons vu, la traduction avec un emploi effréné de l'amplification? La fin de l'essai de Walter Benjamin, lorsqu'il envisage ce que pourrait être «l'idéal de toute traduction », nous suggère que le traducteur doit être capable de lire entre les lignes de l'original : « littéralité et liberté doivent s'unir dans la traduction sous forme de version interlinéaire ${ }^{24}$. Réflexion qui semble indiquer qu'il existerait, dissimulés entre les lignes de l'original, des sens latents qu'une traduction visant le "pur langage» pourrait faire émerger. Il y aurait ainsi dans le texte ce que Panero appelle, dans son Prologue, à Matemática demente, " una Grieta ", "une fissure » ou « un trou » qu'il serait permis de combler au gré de nouvelles traductions. Or c'est ce que propose la traduction-perversion :

«La Perversión, pues, trabaja en esa Grieta del texto: pero no para agrietarlo, sino precisamente para rellenarlo, perfeccionar, terminar el texto original (una vez más, no para siempre, ya que una nueva traducción, o una simple lectura, encontraría otras Grietas, que llenaran a su vez con nuevas palabras, con nuevos sentidos, viejos por cuanto nacidos - a veces abortados - en el texto original. $»^{25}$

Les amplifications qui farcissent la traduction-perversion auraient donc pour fonction de combler le texte original, qui serait comme inachevé, en exploitant sa virtualité ; des sens latents qui ne demanderaient qu'à émerger au gré de nouvelles traductions, un peu comme si le texte source possédait une sorte de subconscient. Ce que semble justifier la phrase qui conclut l'essai de Benjamin: "La version interlinéaire du texte sacré est l'archétype ou l'idéal de toute traduction $»^{26}$. D'où la démarche extrême et paradoxale de Panero pour qui la perversion est « la única traducción fiel y ello mediante el adulterio, la infidelidad $»^{27}$.

Mais quels sens prend cette pratique de la traduction comme perversion vis-à-vis du sonnet de Bataille ? On a vu que Panero se souciait peu de reproduire la forme canonique du sonnet, forcément dynamitée par de constantes amplifications. Le grand perdant des traductions panériennes, c'est le cadre sonore, voire rythmique de l'original et la façon dont

\footnotetext{
22 Ibid., p. 259.

23 BATAILLE, Georges, «Lo que por fin dijo Benjamin», préface servant à justifier sa traduction des limricks d'Edward Lear, dans Ómnibus, sin sentido, op. cit. Cette citation de Panero très proche de Benjamin est citée en note par Túa Blesa dans «Teoría y práctica de la traducción como perversión », in Traducciones/ Perversiones, op. cit., note 23, p. 24.

${ }^{24}$ BENJAMIN, Walter, La tâche du traducteur, in CEuvres I, op. cit., p. 261.

${ }_{25}$ Matemática demente, op. cit., p. 18-19.

${ }^{26}$ BENJAMIN, Walter, La tâche du traducteur, in CEuvres I, op. cit., p. 262.

${ }^{27}$ Matemática demente, op. cit, p. 17.
} 
le son et le sens y sont indissociables au sein de la signifiance. Par ailleurs, cette traduction fait perdre au texte bataillien sa fulgurance, trait que l'on retrouve dans les autres poèmes de L'Archangélique même s'ils ont des vers libres non rimés. Le procédé systématique de l'amplification tout en suggérant de nouvelles ouvertures alourdit le poème bien qu'elles en garantissent aussi la poéticité par leurs richesses. Mais, si cette traduction demeure aussi un poème grâce à ce renouveau d'images inédites, elle ne saurait être un sonnet. Et il n'est, d'ailleurs, pas inintéressant de constater que le corpus français panérien (Bataille et Corbière) n'est constitué que de sonnets volontairement défigurés par Panero. L'écriture de Panero fondée essentiellement sur la destruction et la transgression non seulement ne peut s'accommoder d'un tel genre mais semble prendre un malin plaisir à le dénaturer. Cependant, en ce qui concerne la traduction du poème bataillien, nous avons pu constater que le vers-librisme n'y est pas vraiment prépondérant - contrairement à d'autres traductions-perversions -, car les vers jouent, ici, avec l'alexandrin et se jouent de lui mais en le soumettant souvent à quelques distorsions dues à des excroissances lexicales ou métriques faisant de sa traduction un monstre.

En fait, ce qui intéresse surtout Panero, c'est la possibilité d'un texte de se faire et de se défaire en proposant des variantes, ce que lui-même propose au sein de Narciso entre el último acorde las flautas, avec un "haiku» (Variable) ${ }^{28}$ de son propre cru présenté avec ses trois variantes ou encore avec le poème «Da-Sein » suivi de «Da-Sein (2da versión) » ${ }^{29}$. Dans un autre poème, dédié à Claudio Rodríguez, ce sont quelques vers qui reçoivent des notes de bas de page indiquant de possibles variantes. Tous ces procédés étudiés et classés par Túa Blesa dans son essai Logofagias. Los trazos del silencio ${ }^{30}$, se réfèrent à des textes qui supposent un vide ou un trou qu'il s'agit de combler. Si bien que l'on ne saurait ignorer le sens que joue aussi ici le motif obsédant pour Panero du «negro agujero » qui justifie à lui seul l'amplification. Il est, d'ailleurs, significatif que Jenaro Talens ait nommé Agujero llamado nevermore $^{31}$ une volumineuse anthologie consacrée à Panero, le vide auquel fait allusion le titre étant inversement proportionnel à l'abondance des textes comme si justement, Panero, le poète le plus prolifique de sa génération, ressentait constamment le besoin de combler un trou.

Plus enclin à rejeter toute explication conceptuelle et à m'en tenir à un empirisme évasif, je souhaitais aborder le cas de la traduction-perversion d'une manière plus en rapport avec la praxis qu'avec la théorie. Mais, tel n'est pas le cas de Panero, qui tente, lui, de se justifier en proposant une théorie de la traduction qui renverrait au bout du compte à l'alchimie ainsi qu'à une sexualité mystique. Et Túa Blesa d'en déduire :

la práctica de la traducción como perversión no es ya tanto un quehacer técnico, sino que se sitúa en una dimensión místico-alquímica, libidinosa, trabajo de escritura del goce y para el goce, que habría de ser liberador. ${ }^{32}$

À part le fait de constater que c'est une affaire liée au "plaisir du texte», à ses « dessous » cachés auxquels Panero juge bon de donner une valeur mystico-tantriste, plaisir qui doit motiver une liberté créatrice inhabituelle du traducteur vis-à-vis du texte source, je n'aurai rien à ajouter et ne souhaite pas aborder l'aspect ésotérique de cette poétique.

\footnotetext{
${ }^{28}$ Narciso en el acorde último de las flautas, in Poesía Completa (1970-2000), op. cit., p. 173-174.

${ }^{29}$ Ibid., p. $175-179$.

${ }^{30}$ BLESA, Túa, Logofagias. Los trazos del silencio, Zaragoza, Colección Trópica, 5. Anexos de Tropelías, 1998.

31 PANERO, Leopoldo María, Agujero llamado Nevermore (Selección poética, 1968-1992), edición de Jenaro Talens, Madrid, Letras Hispánicas, Cátedra, 1992.

32 BLESA, Túa, Teoría y práctica de la traducción como perversión, in Traducciones/ Perversiones, op. cit., p. 29.
} 
En revanche, le choix de Bataille par Panero et ce qu'en a fait sa traductionperversion mérite que l'on en tire certaines conclusions. Chez Bataille l'érotisme est une écriture qui pratique à la fois la transgression et la violence exactement comme chez Panero. Cette écriture-limite est étroitement liée chez eux à la mort et à l'angoisse que celleci suscite. Or, chez Bataille, l'angoisse de mort y est compensée ou neutralisée par un érotisme qui tend constamment vers l'obscène. À défaut de pouvoir révéler le mystère qui suit la mort, Bataille met à nu ce qui ne doit pas être vu, l'obscène. Or, la traductionperversion du sonnet bataillien qui fait de celui-ci un monstre ${ }^{33}$ et casse son cadre très harmonieux a justement la volonté de le tirer vers l'obscénité et son caractère obsédant. Cette traduction-perversion ne parvient-elle donc pas à rendre ce poème encore plus bataillien qu'il ne l'était? Comment pourrait-il en être autrement quand l'œuvre poétique bataillienne suppose, elle-même, la notion de perversion par rapport à la poésie, comme nous l'explique si bien Bernard Noël qui écrit à propos d'elle :

Elle représente un danger pour la poésie. Elle n'en conteste pas seulement les manières, elle les déchire, les salit ou bien les rend dérisoires. Ainsi la poésie est attaquée dans sa nature même et bientôt pervertie ou, plus exactement souillée. ${ }^{34}$

Tout cela s'applique parfaitement à la traduction-perversion de Panero par rapport au sonnet bataillien. Grâce au motif récurrent du «negro agujero » lié à l'œil, la traduction panérienne retrouve l'obscène et l'obsédant qui caractérise la violence outrancière des récits de Bataille à laquelle échappe curieusement ce sonnet écarté de L'Archangélique par son auteur. Agathe Simon, dans un article intitulé justement «L'obscène et l'obsédant ", signale que le plus souvent le personnage bataillien évite l'orgasme pour demeurer le plus longtemps possible dans «cette tension perpétuelle tout juste contenue et volontairement entretenue, tandis que l'éblouissement instantané est sans cesse évité ${ }^{35}$. Or, nous avons constaté que si le premier tercet du sonnet bataillien dit sous la forme d'une gradation ascendante et au passé simple cet «éblouissement» orgasmique, la traduction panérienne préfère, elle, la rapporter sans réelle gradation comme un événement à venir, encore non advenu comme le montre l'usage du futur «un resplandor inmenso entonces me iluminará ». Cette «mutación de Bataille » est donc comme une façon de rappeler à Bataille ce qu'il est. Aidé sans doute par une excellente connaissance de l'œuvre bataillienne, Panero nous donne ainsi sa propre « version interlinéaire » du sonnet, et pour ce devient lui-même obscène en révélant l'envers du secret.

\footnotetext{
33 Il convient d'observer que Panero lui-même revendique le terme de «monstre» pour parler de ses traductions. Ainsi déclare-t-il dans sa préface à sa traduction de «The hunting of the Snark », in El ómnibus, sin sentido, op. cit., : «Porque traducir es pervertir, es, de algún modo, crear monstruos ». p. 280.

${ }^{34}$ NOËL, Bernard, «Le bien du mal », préface à son édition de L'Archangélique et autres poèmes, op. cit., p. 7.

${ }^{35}$ SIMON, Agathe, « Georges Bataille : l'obscène et l'obsédant », La voix du regard, n 15 , automne 2002, p. 21.
} 